\title{
Rapid Fabrication of Bio-inspired Antireflection Film Replicating From Cicada Wings
}

\author{
Ze Wang ${ }^{1}$, Bo Li ${ }^{1,2}$, Xiaoming Feng ${ }^{1}$, Zhibin Jiao ${ }^{1}$, Junqiu Zhang ${ }^{1,3,4}$, Shichao Niu ${ }^{1,4^{*}}$, \\ Zhiwu Han ${ }^{1 *}$, Luquan Ren ${ }^{1}$ \\ 1. Key Laboratory of Bionic Engineering (Ministry of Education, China), Jilin University, Changchun 130022, China \\ 2. Department of Chemistry, University of California-Riverside, California 92521, USA \\ 3. Department of Mechanical Engineering, Columbia University, New York 10027, USA \\ 4. State Key Laboratory of Automotive Simulation and Control, Jilin University, Changchun 130022, China
}

\begin{abstract}
Inspired by cicada wings, a flexible film with self-cleaning and broadband antireflection properties was fabricated with a rapid, straightforward and cost-effective method. The cicada wing was selected as the original template, and a polymethyl methacrylate (PMMA) negative replica was obtained by evaporation solvent process. The original template was directly peeled off. Subsequently, the polydimethylsiloxane (PDMS) was spread in the as-prepared PMMA negative replica. After curing and peeling processes, the PDMS positive replica was manufactured successfully. The morphologies and performances of cicada wings were perfectly inherited by the PDMS positive replica. What is more, the excellent optical property of cicada wing was investigated experimentally and theoretically. Compared with flat PDMS film, the average reflectivity of structural PDMS film was reduced from $9 \%$ to $3.5 \%$ in the wavelength range of $500 \mathrm{~nm}-$ $900 \mathrm{~nm}$. These excellent antireflection properties of bio-inspired antireflection film can be attributed to the nanostructures which achieve a gradient refractive index between air and the materials, and the mechanism of the antireflection properties was revealed via effective medium theory. Besides, the bio-inspired broadband antireflective film exhibited superhydrophobic property after the surface treatment (a $152.1^{\circ}$ water contact angle), and it also displayed satisfactory flexibility. This work provided a universal method to fabricate the exquisite biological structures, realizing the transfer of structure and function. Moreover, the multifunctional antireflection film exhibited the potential value for applications in optical communications, flexible display screens, and anti-dazzle glasses.
\end{abstract}

Keywords: antireflection, self-cleaning, multifunctional surface, flexible film, biomimetic fabrication

Copyright $($ The author(s) 2020 .

\section{Introduction}

Along with networks widely applied, the mobile phone and various electronic displays are tied up with our daily life. However, the troubles arise when they are used outside or in strong light surroundings. The intense light glowing out from the surface not only would impair the vision, yet the information we want is disturbed. Besides, the glare on automobile mirror and windshield is becoming a potential danger for safe driving. What's worse, strong reflection on the optoelectronic conversion device will lose a lot of light energy, reducing the energy conversion efficiency ${ }^{[1-4]}$. Hence, the dazzling light is supposed to be suppressed, and it's also a hot topic for researchers. As is known to all, the strong reflection is caused by the sudden change of the refractive index in different media, and that is "Fresnel reflection", it is important to achieve antireflection by depressing the Fresnel reflection ${ }^{[5-7]}$.

At present, there are two ways to reduce reflection. One is to fabricate multi-layer interference coating ${ }^{[8-12]}$, and the other is prolonging the optical path to achieve light-trapping effect ${ }^{[13-15]}$. However, the limitation of the former is that the mechanical robustness is not satisfactory, and the latter shows poor transparency and obvious scattering. Therefore, current techniques are not suitable for applications such as the surface of displays. In brief, an optical surface with high transmittance and low reflectivity needs to be studied. Since the properties of the antireflective surface are closely related to its materials and structures on it. It is important to select proper materials and structure parameters.

Inspired by nature, many bionic functional surfaces with low energy consumption and high adaptability have

\footnotetext{
*Corresponding author: Shichao Niu, Zhiwu Han
}

E-mail: niushichao@jlu.edu.cn, zwhan@jlu.edu.cn 
been investigated. For example, the vibration perception of nocturnal scorpion ${ }^{[16]}$, the bright structural colors on beetle back and butter fly wings ${ }^{[17-20]}$, the antireflection performance of the moth eye and cicada wing ${ }^{[21-24]}$, dynamically adjustable skin color and reflectivity in squid and other cephalopods ${ }^{[25,26]}$.

Learning from nature, creatures with anti-reflective properties have been selected. The typical cases for antireflection are cicada wing and moth eye. Although they both have periodic nanostructures, yet the morphology of the former is closer to hemispherical shape and that of the latter is similar to the cone with the dome. The advantage of cicada wing in size makes it more suitable as the prototype for investigation. Since the unique properties of these biomaterials are closely related to the microstructures covering on their surfaces. These nanostructures are supposed to be experimentally and theoretically investigated in detail. Meanwhile, in order to inherit the exquisite structure parameters of the prototype, the organism would be used as the template.

Here, the cicada which has transparent wings with remarkable self-cleaning properties and high transmittance over the whole visible spectral range, was chosen as the bionic prototype. The antireflection mechanism of the natural cicada wing was also studied in detail. At the same time, the nanostructures of natural cicada wing were transferred to polymer material successfully, which realized the perfect inheritance of morphology and performance of nanostructures covering on cicada wing. What's more, it might be helpful to shorten the gap between bio-inspired theoretical design and practical applications.

\section{Materials and method}

\subsection{Periodical nanostructures in original cicada wings}

The wings of cicada sp12 were chosen as biological specimen used for further experiment. It's a common cicada from Qingmai, belonging to the uncertain species under cicada. These cicadas are named after the Latin name "sp12", and the cicada specimens were provided by Shanghai Qiu Yu Biotechnology Co., Ltd. The cicada wing was thin and transparent. Meanwhile, there were some supporting networks on the surface. As shown in Fig. 1a, the glare on the glass slide was caused by the mirror reflection, which has interfered the readability of the words. As for the cicada wing, this situation is completely avoided. It's obvious that the natural cicada wing possesses satisfactory antireflection performance. Besides, some drops were added on the cicada wing to observe its wettability, and a $138.8^{\circ}$ water Content Angle (CA) was measured with the help of water drop angle measuring instrument (KRUSS DSA25) (Figs. 1b and 1c). Additionally, the reflection spectrum and transmittance spectrum of the cicada wing were obtained by a spectrometer (Ocean Optics USB 4000) in Fig. 1i. It could be found that the cicada wing had high transparent $(>80 \%)$ and low reflectivity $(<3 \%)$ in the visible light region.

Since the function was closely related to the structures covering on the surface of the creature, the cicada wing was observed with Field Emission Scanning Electron Microscope (FESEM) in Figs. 1d - 1f. It could be found that the thickness of the wing was about $6.25 \mu \mathrm{m}$, and the nanostructures seen from front and back of the wing were almost the same. Besides, the morphology of the structures looked like the parabolic shape which has been proved to be the best one in the field of antireflec$\operatorname{tion}^{[27,28]}$. Its structure parameters including the period, the diameter of the base and the height were $170 \mathrm{~nm}$, $170 \mathrm{~nm}$, and $280 \mathrm{~nm}$, respectively. At the same time, the nanostructures with a tight hexagonal arrangement were characterized with Atomic Force Microscope (AFM), the three different curves in Fig. 1h meant the configuration of the surface at different cross-sections which were orthogonal to the surface of the cicada wing. It indicated that the differences between different regions were tiny, and the arrangements of nanostructures on the surface of cicada wing were uniform.

\subsection{Antireflection mechanism of the nanostructures on cicada wings}

Fig. 2 is used to illustrate the mechanism of antireflection. Multiple optical behaviors occur on the surface of the cicada due to the presence of the surface nanostructure (Fig. 2a). For sub-wavelength structures, the incident wavelengths are equivalent to their sizes, and the light interacts with the entire surface. Here, the nanostructures distributing on the cicada wing and the air filled between the nano structures are treated 

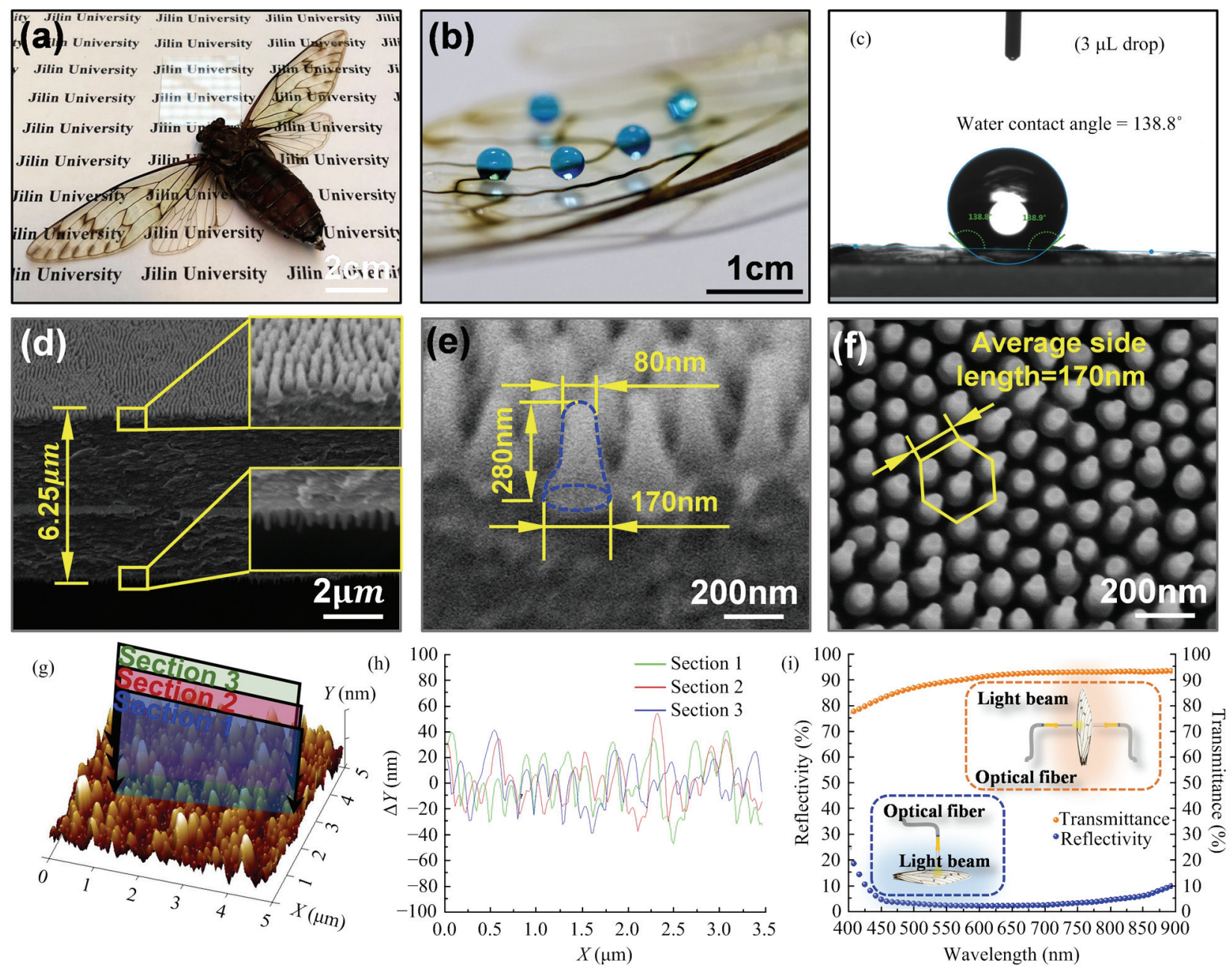

Fig. 1 The structures and excellent properties of natural cicada wings. (a) The glass slide and cicada specimen putting on the paper; (b) the drops on the cicada wing; (c) the static water contact angle on the surface of the cicada wing; (d) - (f) the FESEM microscope images of the cicada wing observed from the side view and top view; (g) the AFM image of the cicada wing; (h) the profiles of different sections on cicada wing; (i) the reflectivity spectrum and transmittance spectrum of the cicada wing.

(a)

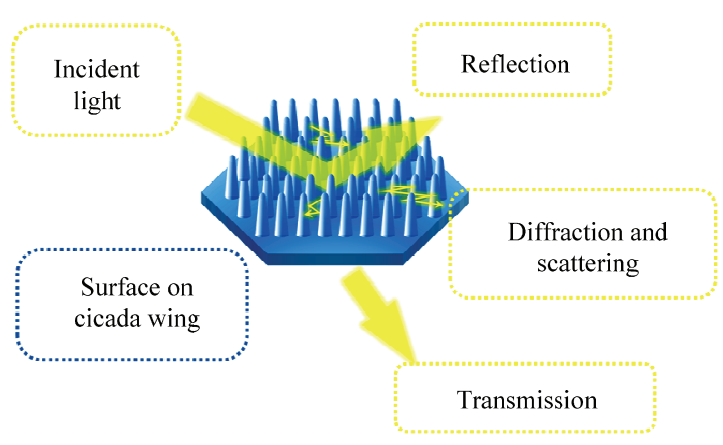

(b)

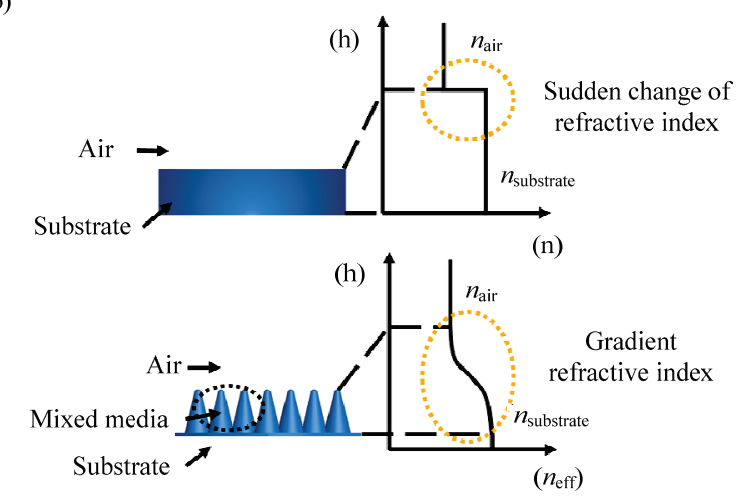

Fig. 2 Schematics of mechanism for antireflection of the cicada wing. (a) Various optical behaviors on the surface of cicada wings; (b) the diagram of the gradient refractive index. 
as mixed media. The surface structure can be considered as a superposition of films with continuous variation of refractive index. According to the theory of effective medium $^{[29]}$, it could be expressed as:

$$
\frac{n^{2}-n_{1}^{2}}{n^{2}+2 n_{1}^{2}}=\left(1-f_{1}\right) \frac{n_{2}^{2}-n_{1}^{2}}{n_{2}^{2}+2 n_{1}^{2}} .
$$

Here, the $n$ is the effective refractive index of adjacent dielectric layers. $n_{1}$ and $n_{2}$ is the refractive index of different materials. If $n_{1}$ is exchanged by $n_{2}$, which means the layer of $n_{1}$ is surrounded by the layer of $n_{2}$, the resultant $n$ will be changed. $f_{1}$ is the proportion of air in the mixing media. Besides, according to the Bruggeman Effective Medium Approximation (EMA) ${ }^{[30]}$, the "effective medium" is assumed to be a homogeneous mixture of two constituent layers, which yields:

$$
f_{1} \frac{\left(n_{1}^{2}-n^{2}\right)}{\left(n_{1}^{2}+2 n^{2}\right)}+f_{2} \frac{\left(n_{2}^{2}-n^{2}\right)}{\left(n_{2}^{2}+2 n^{2}\right)}=0 .
$$

The surface of the cicada wing can be regarded as the superposition of numerous thin films with continuously varying refractive index because of the continuous outline of nanostructures. The uniqueness of EMA is that it can be extended to multiple numbers of constituent layers ${ }^{[31,32]}$.

$$
\sum_{i=1}^{n} f_{i} \frac{\left(n_{i}-n^{2}\right)}{\left(n_{i}^{2}+2 n^{2}\right)}=0 .
$$

Therefore, the equivalent refractive index of cicada-air mixed media can be expressed as ${ }^{[33,34]}$.

$$
n_{e f f}=\sqrt{n_{a}^{2} \cdot f+n_{s}^{2} \cdot(1-f)} .
$$

Here, $n_{\text {eff }}$ is the effective refractive index of mixed media, and $f$ refers to the proportion of air and substrate materials in different positions. Besides, $n_{a}$ is the refractive index of air, as well as $n_{s}$ is the refractive index of the substrate. The $h-n_{\text {eff }}$ curves of the flat surface and structural surface are expressed respectively (Fig. 2b).

As shown in Fig. 2b, when the flat surface is illuminated by light, Fresnel reflection occurs due to the difference of refractive index between the substrate and the air. However, the existence of nanostructures on the surface of cicada wings can be approximately a group of multi-effective dielectric layers with gradient refractive index from air to substrate. In conclusion, it can be found that the sudden change of the refractive index has been smoothed by the nanostructures. Therefore, Fresnel reflection is suppressed and the antireflection on cicada wing is achieved.

\subsection{Biomimetic fabrication}

\subsubsection{The source of reagents}

Ethanol, acetone, and N-Hexane are provided by Beijing Chemical Works (analytical reagent), deionized water was provided by Sangon Biotech (Shanghai) Co., Ltd. Methylbenzene was provided by Sheng Tongyue Chemical Industry (analytical reagent), and pulverous PMMA was provided by Sumitomo Chemical. Polydimethylsiloxane (PDMS, Sylgard 184) was provided by Dow Corning.

\subsubsection{Pretreatment}

First, the wing was cut to eliminate the fibrous support network and some parts of the wing edges. Then, the wings were pretreated with acetone and ethyl alcohol for $10 \mathrm{~min}$ respectively to remove the impurities attached to the surface. After that, the biological specimens were kept dry naturally.

\subsubsection{PMMA negative replica}

Figs. $3 a-3 c$ show the fabrication of the PMMA negative replica. Before the experiment, the powdered PMMA was pretreated under $70{ }^{\circ} \mathrm{C}-80^{\circ} \mathrm{C}$ for $2 \mathrm{~h}-4 \mathrm{~h}$ to remove the moisture. Then the powdered PMMA and methylbenzene (7: 100 in weight) were stirred by a magnetic stirrer under $60{ }^{\circ} \mathrm{C}$ for $30 \mathrm{~min}$, forming a completely transparent colloidal mixture. In this process, heating can facilitate the dissolution process, but not necessary. After that, the cicada wing was used as an original template, then the mixture of the PMMA and methylbenzene was added on it. Subsequently, the cicada wing was moved into a vacuum drying oven under $45^{\circ} \mathrm{C}$ to evaporate the solvent. After removing the cicada wing, a thin and transparent PMMA film with negative structures was fabricated.

\subsubsection{PDMS positive replica}

Figs. $3 d-3 f$ show the fabrication of the PDMS positive replica. First, the N-Hexane was added to the PDMS monomer, and the proper proportion of 
N-Hexane in PDMS monomer was about $8 \%$. Then the hardening agent was evenly mixed with the monomer with 10: 1 ratio in weight. Here, the N-Hexane was used to improve the mobility of the mixture. Next, the mixture was injected into the PMMA negative replica and followed by a heat solidification under $0.08 \mathrm{MPa} 80{ }^{\circ} \mathrm{C}$ for 2 h. At last, taking the thin film off carefully, and the antireflection film was obtained.

\section{Results and discussion}

\subsection{Structure characterization}

The morphologies and structures of PMMA negative replica and PDMS positive replica were characterized by Atomic Force Microscope (AFM, BRUKERDIMENSION FASTSCAN) and Field Emission Scanning Electronic Microscopy (FESEM, JEOL JSM$6700 \mathrm{~F})$. The elements and functional groups were characterized with the help of Energy Dispersive Spectroscopy (EDS, OXFORD INSTRUMENTS X-MAX) and Fourier infrared spectroscopy (FTIR, SHIMADZU IRAffinity-1S) respectively.

\subsubsection{Structure characterization of PMMA negative replica}

Fig. 4a show the solidified PMMA negative replica covering on the cicada wing, and the nanostructures distributing on it were displayed in the dotted line area. In order to further investigate the structure, the FESEM images of the PMMA negative replica were shown in Figs. $4 \mathrm{~b}-4 \mathrm{c}$. It was obvious that the negative nanostructures looked like nanoporous holes which were similar to the inverted- $V$ shape. At the same time, the parameters including its period, height, and diameter were consistent with the original prototype. Besides, the elements of the PMMA negative replica and cicada wing were analyzed in Fig. 4d. Here, the Au-peak was caused by the gold-spraying process in order to increase the conductivity of the sample, so it would not be considered. The spectra in dark blue and baby blue matched with the PMMA negative replica and cicada wing respectively. It was obvious that the components of PMMA negative replica were carbon $(70.12 \%$ in atomic percentage) and oxygenium $(29.88 \%$ in atomic percentage). However, the spectrum of the prototype in red dotted line magnification diagram contained an obvious $\mathrm{N}$-peak which was different from the PMMA. It indicated that the PMMA negative replica and the prototype could be separated completely and there was hardly any residue left.

\subsubsection{Structure characterization of PDMS positive rep-} lica

The digital photograph in Fig. 5a show the PDMS positive replica. The nanostructures on its surface were shown in the schematic diagram in the dotted line area. Figs. $5 \mathrm{~b}$ and $5 \mathrm{c}$ indicated that the nanostructures on the cicada wing were inherited perfectly by the PDMS positive replica. It could be seen that the height of the nanocone was about $230 \mathrm{~nm}$, which was slightly lower than that of the original prototype and the negative replica. On the one hand, this might be caused by the limitation of the wettability of the material, on the other hand, there was a certain volume reduction phenomenon in the curing process. In general, the features of the original morphologies of the prototype were transferred to the positive PDMS replica. Besides, the functional group

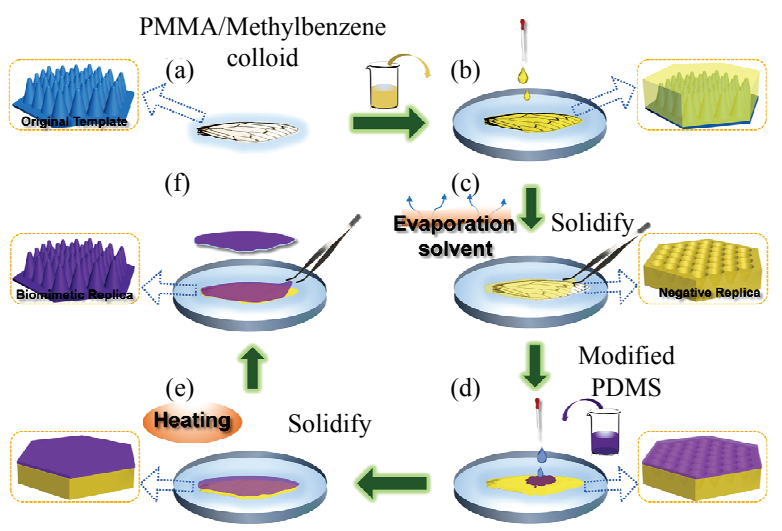

Fig. 3 The schematic drawing of biomimetic fabrication. (a) - (c) shown the process of rebuilding the biomimetic negative replica; (d)-(f) shown the process of rebuilding biomimetic positive replica.

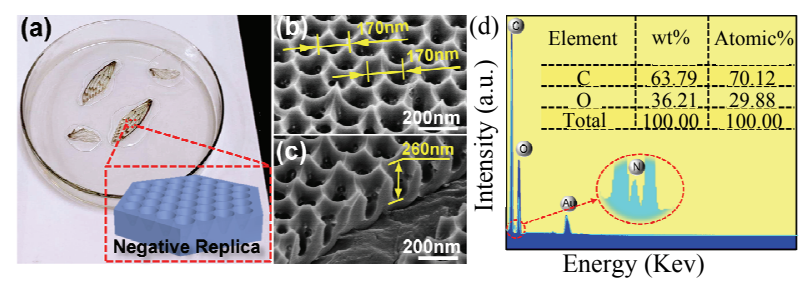

Fig. 4 The morphologies and components of the PMMA negative replica. (a) The PMMA solidified on the surface of cicadas wings; (b) - (c) the FESEM images of the PMMA negative replica; (d) the EDS spectrum of PMMA negative replica and cicada wing. 

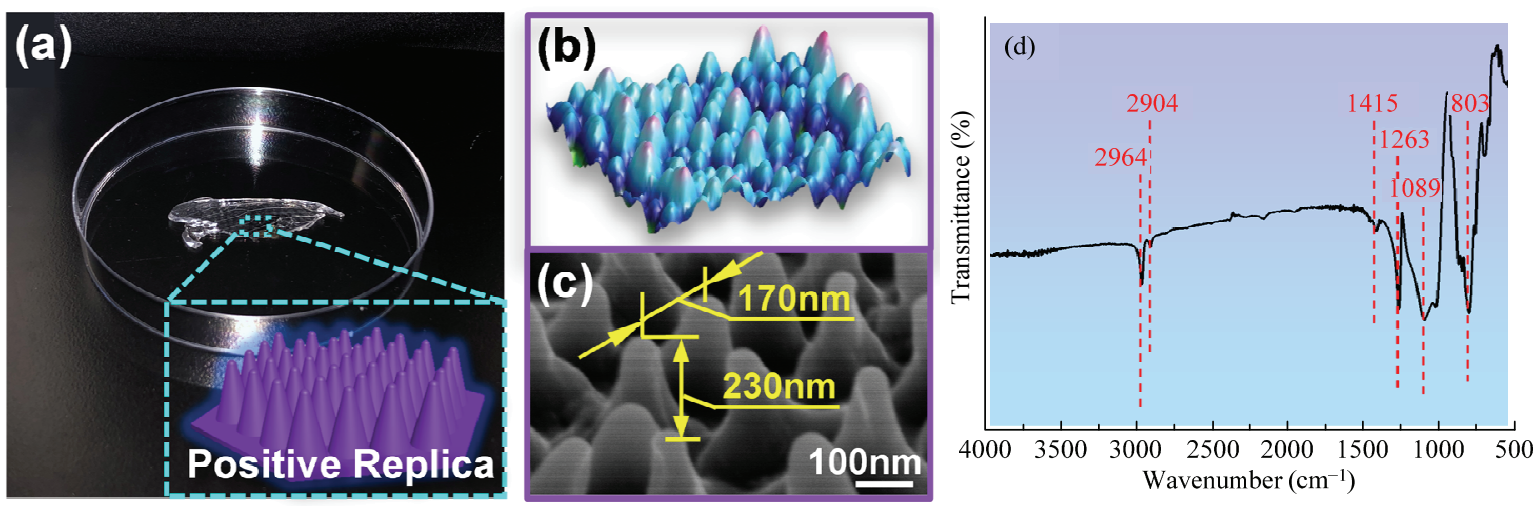

Fig. 5 The morphologies and components of the PDMS positive replica. (a) Digital photograph of PDMS positive replica; (b) the AFM image of PDMS positive replica; (c) the FESEM image of PDMS positive replica; (d) the FTIR spectrum of PDMS positive replica.

characterization of the positive structural film was carried out with the help of Fourier infrared spectroscopy, and the result in Fig. 5d illustrated that the material of positive PDMS replica was PDMS.

\subsection{Optical and self-cleaning performance of the replica}

The optical and self-cleaning performances of the biomimetic replica were characterized with the help of a miniature fiber-optic spectrometer (Ocean Optics USB 4000) and water drop angle measuring instrument (KRUSS DSA25).

As shown in Fig. 6a, although the cicada wing was mainly composed of chitin, the reflectivity of the non-structural chitin was much higher than that of the surface of the cicada wing. It indicated that the structure had a great effect on the reflectivity of the surface. Meanwhile, the reflectivity of the biomimetic replica was about $3.5 \%$, and it was much lower than that of flat PDMS. It indicated that the nanostructures on the positive replica played an important role in antireflection. Besides, compared with flat PMMA film, the negative replica also displayed a certain antireflective performance. It was obvious that the cicada wing displayed the lowest reflectivity in the visible region while the PDMS positive replica was slightly inferior. On the one hand, it might be caused by the fact that the height of the nanostructures on positive replica was lower than that of the original structure. On the other hand, the structural configuration between the prototype and prepared replica was quite different. The natural cicada wings assembled nanostructures on both interfaces, while the biomimetic replica only showed structures on one interface. Both of the structural configuration and structure parameters play an important role on antireflection. Therefore, the anti-reflection performance of the replica needed to be further improved by improving the process of biomimetic fabrication, and we will focus on it as the next step in our work in the future.

Considering practical application, transparency was quite important for antireflective films. The transmittance curves of different surfaces were reorganized in Fig. 6b. Compared with the flat PDMS film, the structural PDMS film performed better. Meanwhile, the transmittance of the glass slide was about $86 \%$, and it was improved to $90 \%$ by attaching the biomimetic replica. However, the transmittance of glass slide covered with biomimetic replica was slightly lower than that of single biomimetic replica. Perhaps it was caused by the increase of the whole assembly thickness when the film was attached, and the absorption of light was enhanced. Besides, the bonding quality may not be satisfactory, and there were defects such as small bubbles on the interface which reduced transmittance. In our previous work, the bioinspired film was fabricated with PMMA, and the average transmittance of the single film over the visible region was about $93 \%{ }^{[35]}$. In order to compare the effects of similar structures fabricated with different materials, the results of optical properties were analyzed in Figs. $6 c-6 d$. In contrast, the average transmittance of the PDMS positive replica reached $91 \%$. Although refractive index of the two materials is different 


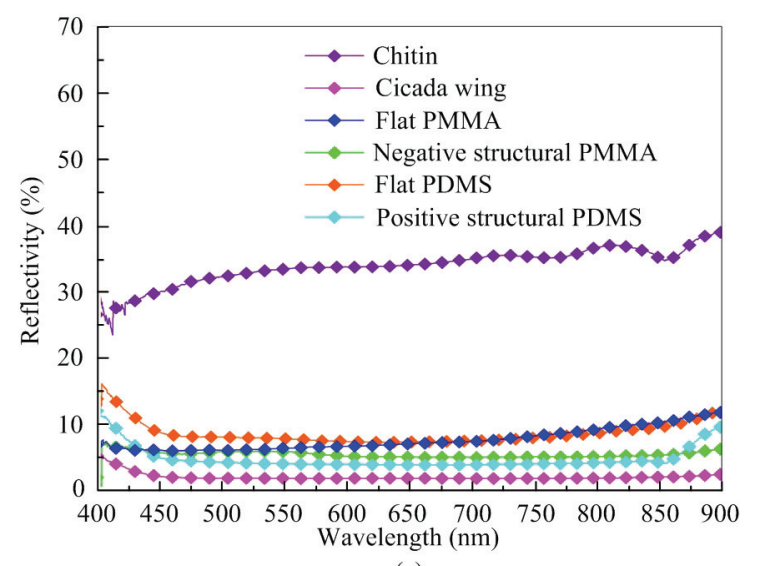

(a)

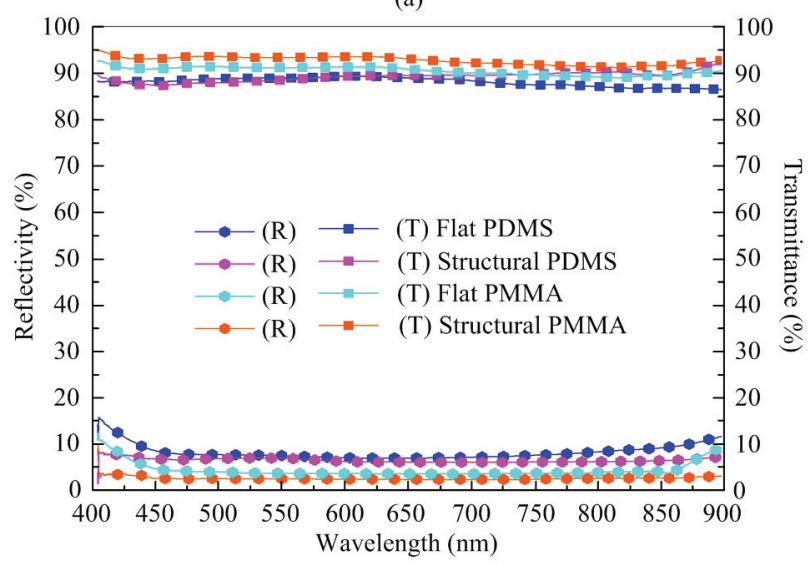

(c)

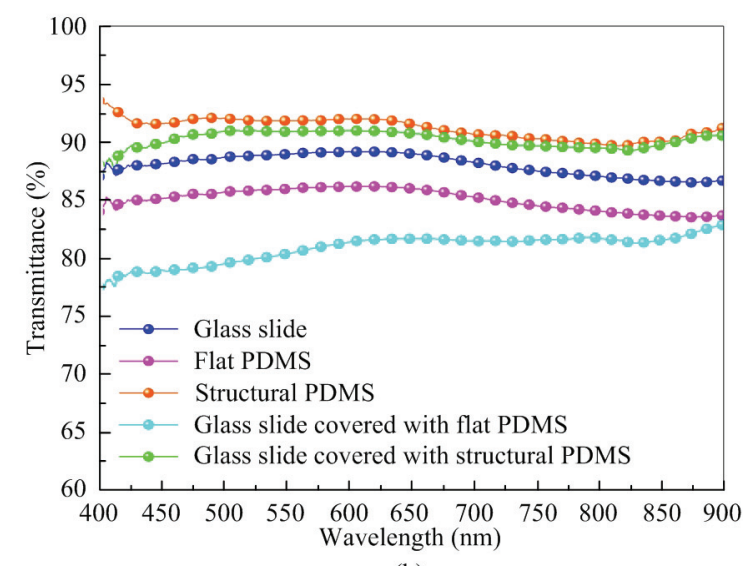

(b)

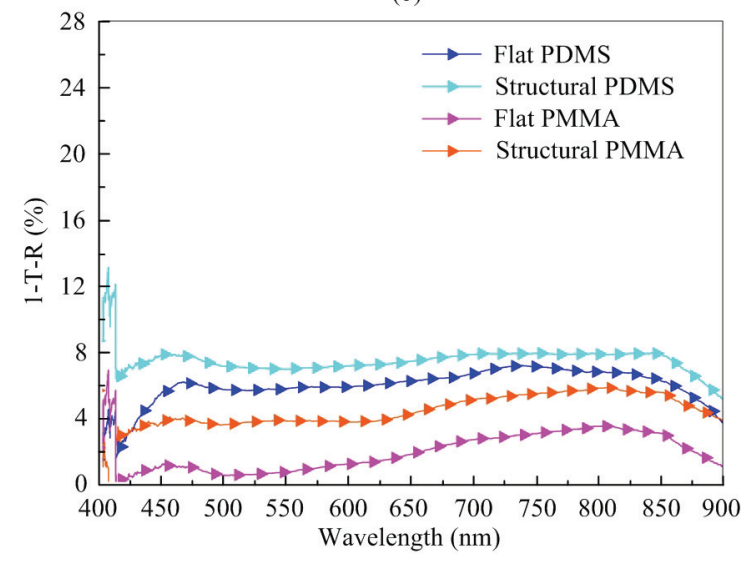

(d)

Fig. 6 The optical performance analysis of the positive PDMS replica. (a) Reflectance spectra of different surfaces; (b) transmittance spectra of different surfaces; (c) optical properties of the films fabricated with different materials; (d) the S (1-T-R) curves of the films fabricated with different materials.

$\left(n_{\mathrm{PMMA}}=1.49, n_{\mathrm{PDMS}}=1.404\right)$, yet the optical performances of replica prepared with PDMS were comparable to that of PMMA. It could be found that the influence of structure was greater than that of material in antireflection. Meanwhile, the film prepared with PDMS was thicker, which had a certain decrease in the transmittance. Therefore, the transmittance of the PDMS positive replica was not as good as that of PMMA positive replica. Besides, the results in Fig. 6d were obtained by 1-T-R, and it was recorded as ' $\mathrm{S}$ ' here. The scattering and absorption between the nanostructures were often included ${ }^{[36]}$. The structural films exhibited obvious increase in $\mathrm{S}$ curve compared with flat films, indicating that the structures promote the scattering of light. Besides, compared with the PDMS replica with a thickness of $1 \mathrm{~mm}$, the PMMA replica with a thickness of $0.18 \mu \mathrm{m}$ was quite $\operatorname{thin}^{[35]}$, and the absorption of the latter was negligible. Therefore, the $\mathrm{S}$ curve of the film prepared with PDMS was universally higher than that of PMMA whether with or without structure. It indicated that the thickness of the film had a great influence on the scattering and absorption.

The existence of the nanostructures could eliminate the sudden change of refractive index. Hence the reflection was depressed. Meanwhile, the sub-wavelength structures were effective to enhance the scattering of the surface. The concentrated light falling on the structural surface could be dispersed by the nanostructure arrays. Due to the scattering in all directions, the light falling to the observation point was weakened (Fig. 7a). Therefore, the structural PDMS replica could display a higher transmittance and lower reflectivity compared with flat PDMS. Fig. 7b displays the replica under strong light. The non-structural region of the replica exhibited an obvious specular reflection in a strong light environment, meanwhile, the glare made it difficult to read the words. 
As a contrast, the words under the structural region were partially readable. Besides, the blurring in structural region was caused by the ridges and the supporting structures covering on the replica. These structures could date back to the network structures on original cicada wing. At present, there is no good way to solve the problem. The structural film may display a more satisfactory antireflection if the ridges and supporting structures were completely rejected.

Flexibility is also one of the evaluating indicators for antireflective surfaces. The prepared structural PDMS replica has shown excellent elasticity and flexibility (Fig. 7b-1). What's more, a stretching test was used to investigate. As shown in Fig. 7c, a homemade equipment to stretch the film, and the extension ratio (ERO) of film was defined as:

$$
E R O=\frac{\left(l_{1}-l_{0}\right)}{l_{0}} \times 100 \%
$$

$l_{1}$ was the length of the film drawn under external force, and $l_{0}$ was the original length of the film. The film was stretched at a certain ERO for $5 \mathrm{~min}$, then it was released. It was observed that the film can restore the original length when the ERO was no larger than $15 \%$. In order to further investigate whether the antireflection performance of the film was affected by the stretch-restore process, the reflectivity curves were measured (Fig. 7d). The reflectivity curves of $5 \%, 10 \%, 15 \%$ (ERO) were similar. It indicated that the antireflection property of the replica was not destroyed under a 15\% ERO. Meanwhile, these curves were a little higher than that of the replica without stretching process. Perhaps it could be attributed to the uneven part of the surface after the treatment. If the film was stretched with a more than $20 \%$ ERO, the nanoarray on the surface would been destroyed, and it could be confirmed with the SEM image in upper right corner in Fig. 7d. The reflectivity of the destroyed region was higher than $20 \%$. It was obvious that, the bionic antireflection film would not fail under the ERO less than $15 \%$, and it exhibited good flexibility and elasticity.

The self-cleaning performance was also very important for antireflective surface. The static contact

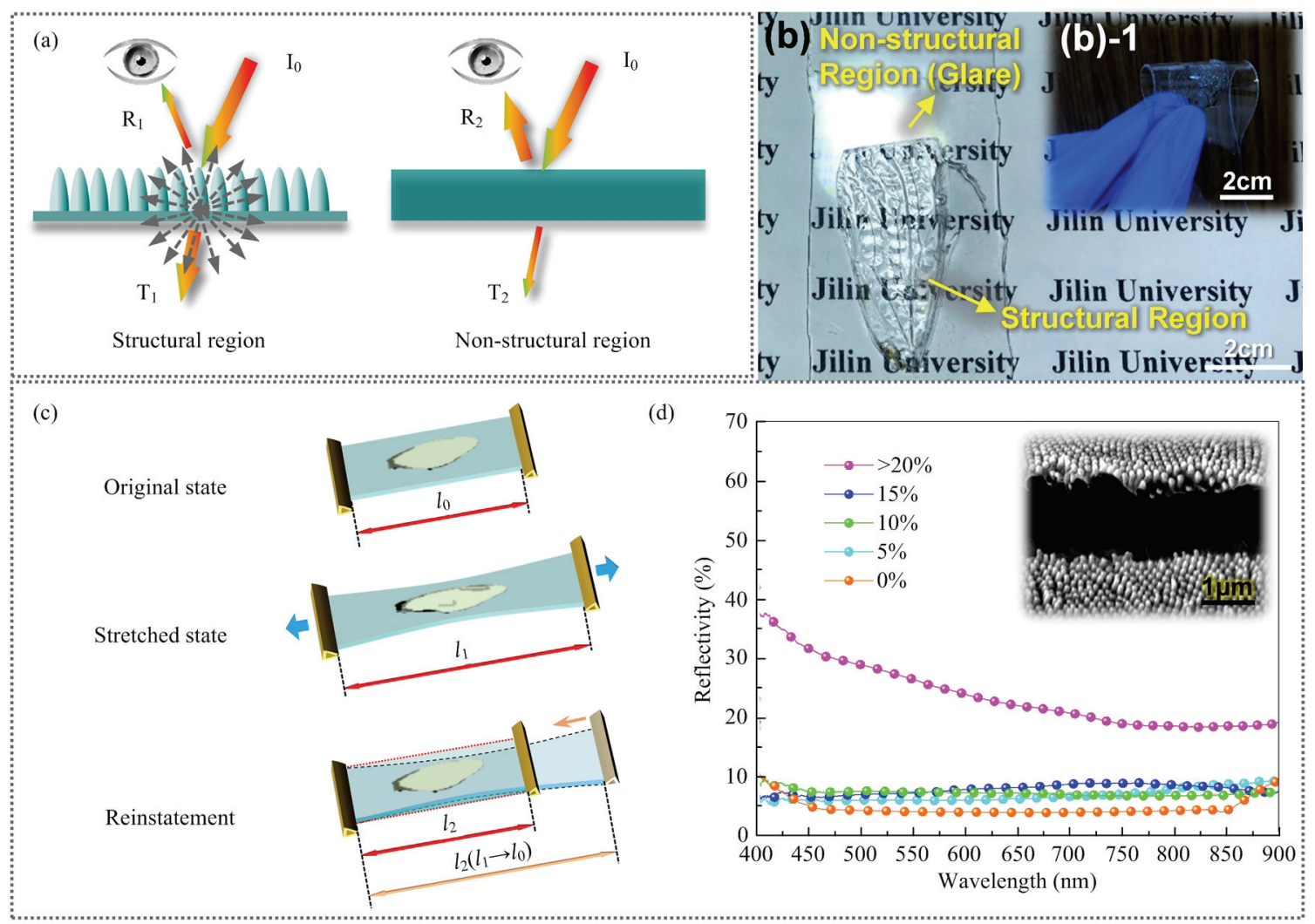

Fig. 7 (a) Schematic diagram of the light pathway through the different regions of the replica; I=Incident light, $\mathrm{T}=\mathrm{Transmission}$, $\mathrm{R}=$ Reflection; (b) the replica under strong light; (b)-1 the replica in a highly curved state; (c) schematic diagram of the stretching test; (d) the reflectivity curves of the PDMS replica after stretch-recovery process. 
angle measurement and dynamic contact angle measurement test were carried out. As shown in Fig. 8a, the contact angles of water droplets on flat PDMS, structural PDMS, and silylanized structural PDMS were measured respectively. The structural PDMS was hydrophobic and reached a $121.1^{\circ}$ Contact Angle (CA), higher than that of flat PDMS. The existence of nanostructures improved the water contact angle of the PDMS surface, indicating that the structure has improved the surface hydrophobicity. After further surface treatment, the contact angle
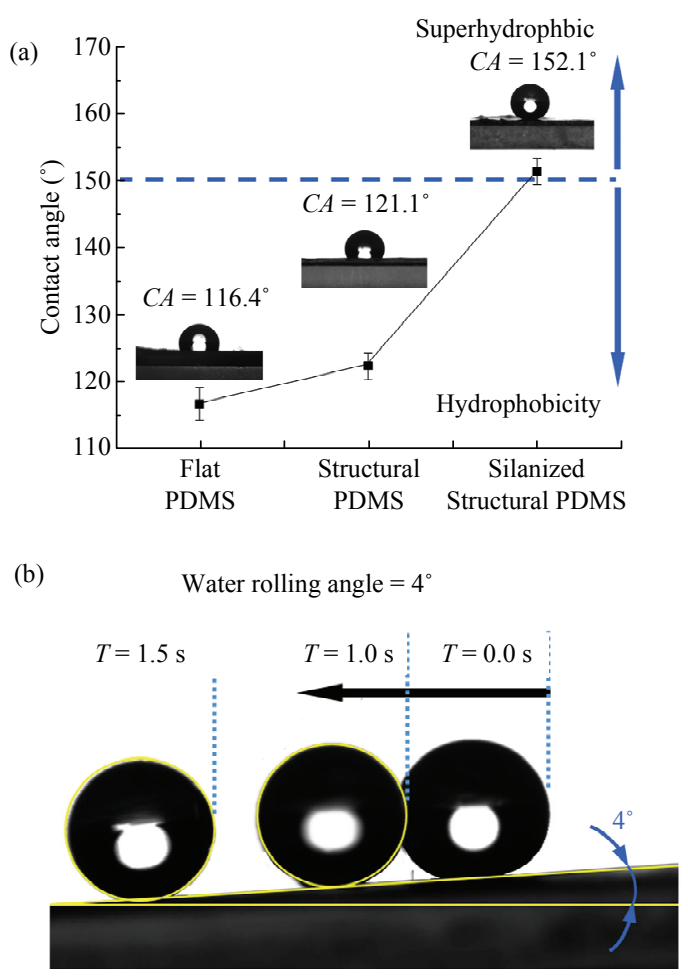

Fig. 8 The self-cleaning performance of the replica. (a) The water contact angle of the flat PDMS, structural PDMS, and silylanized structural PDMS; (b) the water Rolling Angle (RA) of silylanized structural PDMS.
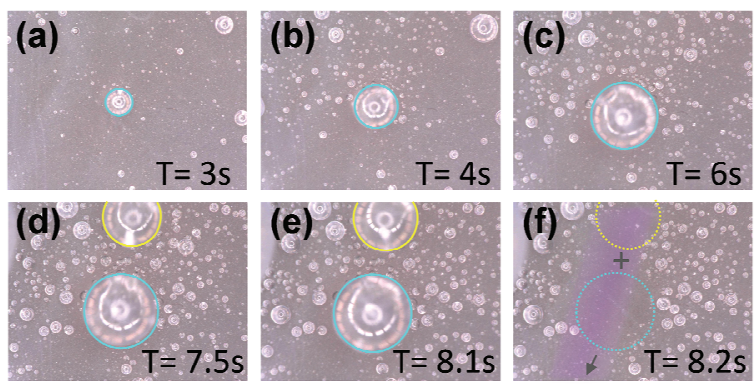

Fig. 9 The positive PDMS replica in fog environment. of the surface reached up to $152.1^{\circ}$, indicating that the surface has reached the superhydrophobic state. In addition, the rolling process was recorded in Fig. 8b, indicating that the adhesion of water droplets on its surface was weak. In summary, the silylanized structural PDMS had good self-cleaning performance.

Since the actual environment was usually complex, it was important for the antireflective film to achieve multifunctional performances. Considering the antireflection in a special environment, such as fog. The results of antifogging performance characterization was shown in Fig. 9. The biomimetic replica was exposed to the spray at room temperature. The fog on the surface grew into small water droplets (Figs. 9a), after that, the water droplets continued to merge with each other and became larger and larger, and finally rolled off on the surface (Figs. 9b-9f), showing a hydrophobic and anti-fogging state.

\section{Conclusion}

In this work, we developed a simple but effective method to mimic the perfect antireflective nanostructures inspired by the cicada wing. Here, the original cicada wing was used as a template to obtain the PMMA negative replica via an evaporation solvent process. Afterwards, the PDMS modified with $\mathrm{N}$-hexane was coated on the surface of PMMA negative replica, followed by a solidifying process. The fabricated structural PDMS film exhibited excellent low reflectivity $(\leq 3.5 \%)$ and high transmittance $(\geq 91 \%)$ in a broadband visible region. By comparing with the previous work, it was also proved that the structure played a greater role in antireflection. Besides, this preparation process made it possible to completely copy the entire cicada wing, which was very important for the research of bionic functional surface. Meanwhile, it was also confirmed that the nanocones were effective for the wettability of the surface. The structural PDMS film achieved the self-cleaning performance after the surface treatment $\left(C A=152.1^{\circ}, R A=4^{\circ}\right)$. Furthermore, its flexibility and antifogging performance were also explored. This work was supposed to come up with a new idea to realize the biomimetic nano-fabrication, and the results in this work were also promised for plenty of practical applications in the fields of curved screens, flexible displays, optoelec- 
tronic devices, solar cells and even anti-dazzle glasses.

\section{Acknowledgement}

This work was supported by the National Key Research and Development Program of China (No. 2018YFA0703300), National Natural Science Foundation of China (Nos. 51835006, 51875244, 51505183, 51325501), JLU Science and Technology Innovative Research Team (No. 2017TD-04), China Postdoctoral Science Foundation Funded Project (2018T110246), Joint Construction Project of Jilin University and Jilin Province (SXGJSF2017-3), and Outstanding Young Talent Fund of Jilin Province (20170520095JH), Scientific and Technological Development Program of Changchun City (Double Ten Project-19SS001), Science and Technology Development Program of Jilin Province (Technology R\&D Project-20190302021GX).

Open Access This article is licensed under a Creative Commons Attribution 4.0 International License, which permits use, sharing, adaptation, distribution and reproduction in any medium or format, as long as you give appropriate credit to the original author(s) and the source, provide a link to the Creative Commons licence, and indicate if changes were made.

The images or other third party material in this article are included in the article's Creative Commons licence, unless indicated otherwise in a credit line to the material. If material is not included in the article's Creative Commons licence and your intended use is not permitted by statutory regulation or exceeds the permitted use, you will need to obtain permission directly from the copyright holder.

To view a copy of this licence, visit http://creativecommons.org/licenses/by/4.0/.

\section{References}

[1] Brongersma M L, Cui Y, Fan S. Light management for photovoltaics using high-index nanostructures. Nature Materials, 2014, 13, 451-460.

[2] Wang W, Zhang J, Che X Z, Qin G G. Large absorption enhancement in ultrathin solar cells patterned by metallic nanocavity arrays. Scientific Reports, 2016, 6, 34219.

[3] Zhou H, Xu J , Liu X H, Zhang H W, Wang D T, Chen Z H,
Zhang D, Fan T X. Bio-inspired photonic materials: Prototypes and structural effect designs for applications in solar energy manipulation. Advanced Functional Materials, 2017, 28, 1705309.

[4] Mahadik D B, Lakshmi R V, Barshilia H C. High performance single layer nano-porous antireflection coatings on glass by sol-gel process for solar energy applications. Solar Energy Materials and Solar Cells, 2015, 140, 61-68.

[5] Lampande R, Kim G W, Park M J, Kang B Y, Kwon J H. Efficient light harvesting in inverted polymer solar cells using polymeric 2D-microstructures. Solar Energy Materials and Solar Cells, 2016, 151, 162-168.

[6] Shao T, Tang F, Sun L X, Ye X, He J H, Yang L M, Zheng W G. Fabrication of antireflective nanostructures on a transmission grating surface using a one-step self-masking method. Nanomaterials, 2019, 9, 180.

[7] Lotz M R, Petersen C R, Markos C, Bang O, Jakobsen M H, Taboryski R. Direct nanoimprinting of moth-eye structures in chalcogenide glass for broadband antireflection in the mid-infrared. Optica, 2018, 5, 557-563.

[8] Saylan S, Milakovich T, Hadi S A, Nayfeh A, Fitzgerald E A, Dahlem M S. Multilayer antireflection coating design for $\mathrm{GaAs}_{0.69} \mathrm{P}_{0.31} / \mathrm{Si}$ dual-junction solar cells. Solar Energy, 2015, 122, 76-86.

[9] Brunner R, Sandfuchs O, Pacholski C, Morhard C, Spatz J. Lessons from nature: Biomimetic subwavelength structures for high-performance optics. Laser and Photonics Reviews, 2012, 6, 641-659.

[10] Anguita J V, Ahmad M, Haq S, Allam J, Silva S R P. Ultra-broadband light trapping using nanotextured decoupled graphene multilayers. Nanotechnology, 2016, 2, e1501238.

[11] Hammond P T. Form and function in multilayer assembly: New applications at the nanoscale. Advanced Materials, 2004, 15, 1271-1293.

[12] Askar K, Gu Z X, Leverant C J, Wang J M, Kim C, Jiang B, Jiang P. Self-assembled nanoparticle antireflection coatings on geometrically complex optical surfaces. Optics Letters, 2018, 43, 5238-5241.

[13] Parnell A J, Bradford J E, Curran E V, Washington A L, Adams G, Brien M N, Burg S L, Morochz C, Fairclough J P A, Vukusic P, Martin S J, Doak S, Nadeau N J. Wing scale ultrastructure underlying convergent and divergent iridescent colours in mimetic Heliconius butterflies. Journal of the Royal Society Interface, 2018, 15, 17425689.

[14] Lee S, Mason D R, In S, Park N. Embedding metal 
electrodes in thick active layers for ITO-free plasmonic organic solar cells with improved performance. Optics Express, 2014, 22, A1145-A1152.

[15] Han Z W, Niu S C, Zhang L F, Liu Z N, Ren L Q. Light trapping effect in wing scales of butterfly papilio peranthus and its simulations. Journal of Bionic Engineering, 2013, 10, 162-169.

[16] Wang K J, Zhang J Q, Fang Y Q, Chen D B, Liu L P, Han Z W, Ren L Q. Micro/nano-scale characterization and fatigue fracture resistance of mechanoreceptor with crack-shaped slit arrays in scorpion. Journal of Bionic Engineering, 2019, 16, 410-422.

[17] He Y D, Zhang Z L, Xue J, Wang X H, Song F, Wang X L, Zhu L L, Wang Y Z. Biomimetic optical cellulose nanocrystal films with controllable iridescent color and environmental stimuli-responsive chromism. ACS Applied Materials \& Interfaces, 2018, 10, 5805-5811.

[18] Rodríguez R E, Agarwal S P, An S, Kazya E, Das D, Shang W, Skye R, Deng T, Dasgupta N P. Biotemplated morpho butterfly wings for tunable structurally colored photocatalysts. ACS Applied Materials \& Interfaces, 2018, 10, 4614-4621.

[19] Zhang Z H, Chen Z Y, Sun L Y, Zhang X X, Zhao Y J. Bio-inspired angle-independent structural color films with anisotropic colloidal crystal array domains. Nano Research, 2019, 12, 1579-1584.

[20] Niu S C, Li B, Mu Z Z, Yang M, Zhang J Q, Han Z W, Ren L Q. Excellent structure-based multifunction of morpho butterfly wings: A review. Journal of Bionic Engineering, 2015, 12, 170-189.

[21] Chan L W, Morse D E, Gordon M J. Moth eye-inspired anti-reflective surfaces for improved IR optical systems \& visible LEDs fabricated with colloidal lithography and etching. Bioinspiration \& Biomimetics, 2018, 13, 041001.

[22] Zeng Y, Chen X F, Yi Z, Yi Y G, Xu X B. Fabrication of p-n heterostructure $\mathrm{ZnO} / \mathrm{Si}$ moth-eye structures: Antireflection, enhanced charge separation and photocatalytic properties. Applied Surface Science, 2018, 441, 40-48.

[23] Xie H, Huang H X, Peng Y J. Rapid fabrication of bio-inspired nanostructure with hydrophobicity and antireflectivity on polystyrene surface replicating from cicada wings. Nanoscale, 2017, 9, 11951-11958.

[24] Zada I, Zhang W, Sun P, Imtiaz M, Abbas W, Zhang D. Multifunctional, angle dependent antireflection, and hydrophilic properties of $\mathrm{SiO}_{2}$ inspired by nano-scale structures of cicada wings. Applied Physics Letters, 2017, 111, 153701.
[25] Levenson R, Bracken C, Bush N, Morse D E. Cyclable condensation and hierarchical assembly of metastable reflectin proteins, the drivers of tunable biophotonics. Journal of Biological Chemistry, 2016, 291, 4058-4068.

[26] Demartini D G, Izumi M, Weaver A T, Pandolfi E, Morse D E. Structures, organization, and function of reflectin proteins in dynamically tunable reflective cells. Journal of Biological Chemistry, 2015, 290, 15238-15249.

[27] Stavenga D G, Foletti S, Palasantzas G, Arikawa K. Light on the moth-eye corneal nipple array of butterflies. Proceedings of the Royal Society B-Biological Sciences, 2006, 273, 661-667.

[28] Boden S A, Bagnall D M. Tunable reflection minima of nanostructured antireflective surfaces. Applied Physics Letters, 2009, 93, 133108.

[29] Chattopadhyay S, Huang Y F, Jen Y J, Ganguly A, Chen K $\mathrm{H}$, Chen L C. Anti-reflecting and photonic nanostructures. Materials Science and Engineering R: Reports, 2010, 69, $1-35$.

[30] Bruggeman D A G. Berechnung verschiedener physikalischer konstanten von heterogenen substanzen. i. dielektrizitätskonstanten und leitfähigkeiten der mischkörper aus isotropen substanzen. Annalen der Physik, 1935, 416, 636-664.

[31] Rytov S M. Electromagnetic properties of a finely stratified medium. Soviet Physics Jetp-Ussr, 1956, 2, 466-475.

[32] Gombert A, Glaubitt W, Rose K, Dreibholz J, Blasi B, Heinzel A, Sporn D, Doll W, Wittwer V. Subwavelength-structured antireflective surfaces on glass. Thin Solid Films, 1999, 351, 73-78.

[33] Jayasinghe R C, Perera A G U, Zhu H, Zhao Y. Optical properties of nanostructured $\mathrm{TiO}_{2}$ thin films and their application as antireflection coatings on infrared detectors. Optics Letters, 2012, 37, 4302-4304.

[34] Braun M M, Pilon L. Effective optical properties of non-absorbing nanoporous thin films. Thin Solid Films, 2006, 496, 505-514.

[35] Han Z W, Wang Z, Li B, Feng X M, Jiao Z B, Zhang J Q, Zhao J, Niu S C, Ren L Q. Flexible self-cleaning broadband antireflective film inspired by the transparent cicada wings. ACS Applied Materials \& Interfaces, 2019, 11, 1701917027.

[36] Ji S, Song K, Nguyen T B, Kim N, Lim H. Optimal moth eye nanostructure array on transparent glass towards broadband antireflection. ACS Applied Materials \& Interfaces, 2013, 5, 10731-10737. 